\title{
Statement of the Technical Group on ECG-VCG and Computer Applications
}

\author{
Meeting of the Research Committee \\ International Society of Cardiology \\ Venice, April 11-17, 1965
}

The quantitative methods and concepts of biophysics can now become a part of the clinical armamentarium. Electrocardiography is particularly amenable to quantitative treatment. The electrical heart source and the field generated by it are subject to the same physical laws as any other current source. Directly applicable and powerful mathematical relationships for treating ECG signals are evolving. Advances in electrocardiography require recognition of these facts with implementation of the quantitative, theorybased approach.

Biophysical contributions to electrocardiography have been made in areas of ECG theory, recording techniques and data handling and analysis. Existing and potential advantages of quantitative methods over current ECG practice are considered here, with areas in which investigation is required.

Contributions to theory:

ECG theory based on sound biophysical principles offers conclusions which restrict or render obsolete several concepts still commonly held in electrocardiography including:

1. The concept of an absolute zero potential to which EGG leads can usefully be referred.

2. The concept of unipolar leads as distinct from bipolar leads.

3. The ventricular gradient as a physiologically-based concept rather than an empirically-bascd time integral of potential.

4. The concept that frontal plane limb leads I, II, and III or leads derived from them by combinations ( $V_{R}, V_{L}, V_{F}, a V_{R}, a V_{L}, a V_{F}$ ) contain intrinsically more information than any pair of them selected at random. (This does not imply that more than two should not be recorded for reasons of convenience.)

Affirmative biophysical contributions to ECG theory include the important conclusions that 1 ) the entire electrical activity of the heart can be

Subcommittee members present: Henry Blackburn, coordinator, Pierre Duchosal, Noboru Kimura, Pentti Rautaharju, Pierre Rijlant.

Consultants present: Hubert Pipberger, Otto $\mathrm{H}$. Schmitt. 
adequately represented for any surface potential analysis by a distribution of dynamically variable current dipole moments within the heart volume; 2) recording and retrieval of ECG data in a time-coherent form will augment the power of ECG analysis.

\section{Contributions to recording techniques and} recommendations for development in quantitative recording of ECG data

Developments in recording technology necessary for quantitative analysis have been made at each level in the system from the skin-electrode contact to the final analytical write-out, but much work toward improvements is required.

\section{At the skin-electrode contact:}

The electrode should be considered a transducer rather than merely a terminal and its characteristics must be considered in the system design. Desirable characteristics are that the electrode be relatively non-polarizable and that it provide across the entire frequency spectrum involved 1) a low electrical impedance with respect to impedance of the amplifier and any directly connected weighting or central terminal networks (to minimize interference and distortion in the ECG due to loading by the amplifier) 2) adequate driving of the amplifier and 3) stabilization against rapid potential changes commonly associated with motion and skin contact (variable offset potentials).

The practice is discouraged of generating composite leads by driving resistance weighting networks directly from skin electrodes unless highly stable amplifiers having exceptionally high input impedance are used with very high impedance weighting networks. Buffer amplifiers offer more reliable weighted leads without need to load the patients electrically. Improved electrodes are now available, and may be particularly useful for recording during physical activity.

At the recorder:

Operation span: The high voltage input range of operation of tape and other EGG recording instruments should be a round number in the 1 to 10 volt range and should represent an adequate compromise between signals small enough to be interfered with easily and so large as to be difficult to generate with solid state amplifiers. Working levels within this span should be large enough to avoid signal-to-noise ratio problems but small enough to prevent overloading from exceptionally high EGG voltages.

Frequency response:

Specifications such as those prepared by the American Heart Association 
are adequate for conventional analysis from direct writing instruments. However, a wider band response is required for research purposes, especially those concerned with significance of components extending into kilocycle regions. On the low frequency end of the spectrum, it is desirable to use true DC amplifiers with special automatic or semi-automatic means of compensating for inevitable DC offset and drift potential, rather than the use of a resistancecapacitance low frequency cut-off. A standard way of achieving this is not yet available.

\section{Monitoring while recording:}

To insure valid records without excessive noise and to guard against accidental failure to record due to faulty switch positioning, all tape recorders should have the capability for monitoring data off the tape while they are being recorded, without degradation of the record quality or that of the monitored reading.

"Noise":

Noise standards are required which are appropriate to electrocardiographic applications in contrast to those which apply to tape recorders made for the entertainment fields.

Signal-to-noise ratios used to determine the acceptibility of residual noise in recording systems should be based on the ratio between the maximal span of voltage linearly recordable and the span of spontaneously occurring voltage subject to interpretation as noise in the recording. The "voltage decibel" is the most accepted measure for signal-to-noise ratio, the input and output impedances being disregarded or considered equal in making the calculations. $\left(\mathrm{Db}=20 \log 10 \frac{\mathrm{ES}}{\mathrm{EN}}\right)$

Behavior when overloaded:

Overloading characteristics that produce distorted records simulating abnormal ECGs must either be avoided by design or else the instrument must generate an adequate alarm signal whenever overload occurs.

Time error:

Flutter and wow ordinarily produces recording and reading errors both in amplitude and in time. The time errors are not usually corrected by flutter compensation systems of the electronic signal additive type. Time error tolerance of tape transports for analysis of time-coherent records should be held to low time values to restrict scatter. The error should be less than the time fluctuation acceptable for a single time trace.

Beside the time error associated with irregular tape movement, there is an irregular variability of time between channels due to tape skewing and a fixed stagger of channel times associated with tape head position scatter. 
Unless this scatter is carefully controlled, tapes will be read with different head time scatters when read on different instruments; and even playing on the same machine on which recording was done will not eliminate error.

Starl-up time:

The time required for a tape recorder to reach a stable speed should be a fraction of a second and all EGG data recorded during the start-up period should be discarded from the analysis. The start-up time is too slow in most available tape recorders.

"Crosstalk":

The noise level specified for individual recorder channels must not deteriorate by virtue of interference (crosstalk) between recording channels unless this crosstalk is separately specified.

Standardization: tape heads and formats

Because of very wide acceptance and soundness of design, the IRIG family of tape recording formats is recommended to promote intercompatibility of recorder even though the IRIG standards unfortunately are non-metric. Either " single" or " double density" IRIG recording is considered as acceptable and machines are considered superior if they will accept and produce either.

Speeds: Desirable standard tape speeds for original recording are 17/8, $33 / 4,71 / 2$ and 15 inches per sec. although for some special purposes and for scaled speed reading 15/16, 30, 60 and 120"/sec. are also acceptable.

Tape width: $1 / 4^{\prime \prime}$ and $1 / 2^{\prime \prime}$ are the most widely used and are standard in medical applications. $1^{\prime \prime}$ tape is also acceptable where real demand for more than 7 channels justifies its high cost.

\section{Destandardization:}

Intentional destandardization of tape speeds, widths or tracking should be discouraged. It is justified only when significant technical recording improvement is brought about or when certain particular applications make it necessary or desirable.

Nodal system design:

As more elaborate recording systems are developed there is increasing difficulty in maintaining interchangeability between the individual components and so the necessity for additional special interfaces or special attenuation arises. To a large extent this trouble can be minimized if modules of a given system class are designed so that they can be freely connected together without overloading, miscoding, attenuating or otherwise upsetting the system. A nodal design approach is encouraged so that individual units or modules may be assembled at will without redesign of the entire system. This involves standardization of levels of voltage and impedance, and buffering 
units, so that any chosen ECG lead system, resistor network, amplifier, recorder or display may be plugged in.

Guides toward standardization of instrumentation:

This committee endorses the national effort of the American Heart Association Subcommittee now preparing detailed guidelines in the field of electrocardiographic instrumentation. It is recommended that the International Society of Cardiology facilitate communication between national societies undertaking similar work in the hope of coordinating these efforts into international agreements on standards.

\section{Contributions to quantitative analysis}

Modern data acquisition and processing systems have the potential to provide precise and reproducible measurements in plentiful quantities. More accurate representation of events at the heart source and reduced measurement variability must, as in any system, improve discrimination, increase predictive power, and strengthen and broaden conclusions made from the data. Work toward realizing this potential is encouraged.

Several active ECG programs exist utilizing FM tape recording with conversion from these tapes into digital form for subsequent manipulation by computer. Reasonably accurate simulation of the analysis of electrocardiograms in the fashion of the cardiographer has been accomplished. Adequate data are not yet available on the simple repeat variability of ECG measurement and interpretation in this type of program and should be provided. Computer-assisted analysis of selected components of the orthogonal ECG recorded on magnetic tape have achieved satisfactory separation of independently classified normal and abnormal populations as well as prediction of autopsy measurements related to ventricular hypertrophy. This accomplishment along with computer averaging techniques which improve the strength of the ECG signal with respect to the " noise" in exercise records, is impossible for human operator.

Many other programs are in progress and will soon reach the stage of effective analysis.

\section{SUMMARY}

In sum, pioneering accomplishments have been made in the application of biophysical concepts and methods to electrocardiography and there is great promise for their facility and power. It is recommended that the International Society of Cardiology encourage and support the orderly transition into effective application of these new tools. However, it is found that the actual 
evidence for superior diagnosis from these methods is preinlimary and that there remain practical and theoretical impediments to their wider utilization. It is considered essential that the new methods retain the contribution to knowledge of the old and during the transition remain as nearly compatible as possible. In this way experience and familiarity in cardiological practice will develop without reaction and implementation of quantitative methods will be expedited. 